\title{
Does admission acetylsalicylic acid uptake in hospitalized COVID-19 patients have a protective role? Data from the Spanish SEMI-COVID-19 Registry
}

\author{
Francesc Formiga ${ }^{1}$ (1) - Manuel Rubio-Rivas ${ }^{1}$. José María Mora-Luján ${ }^{1}$ - Samara Campos Escudero ${ }^{2}$. \\ Rosa Fernandez Madera Martinez ${ }^{3} \cdot$ Manuel Mendez-Bailón $^{4} \cdot$ Pedro Durán-del Campo ${ }^{5}$. Andrea Riaño Pérez ${ }^{6}$. \\ Francisco-Javier García-Sánchez ${ }^{7}$. José Nicolás Alcalá-Pedrajas ${ }^{8} \cdot$ Sergio Arnedo Hernández ${ }^{9}$. \\ Almudena Hernández Milian ${ }^{10}$. Ana Latorre Díez ${ }^{11}$. Ricardo Gil Sánchez ${ }^{12}$. Ramon Boixeda ${ }^{13}$. Julio Vicente ${ }^{14}$. \\ Begoña Cortes ${ }^{15}$. Carmen Mella Pérez ${ }^{16}$. María Esther Guisado Esparteroo ${ }^{17}$. José López Castro ${ }^{18}$. \\ Santiago Rodríguez Suárez ${ }^{19}$. Jose F. Varona ${ }^{20}$ • Ricardo Gomez-Huelgas ${ }^{21}$ • Jose Manuel Ramos-Rincón ${ }^{22}$ • on \\ behalf of the SEMI-COVID-19 SEMI-COVID-19 Network
}

Received: 24 June 2021 / Accepted: 4 October 2021 / Published online: 29 November 2021

(c) Società Italiana di Medicina Interna (SIMI) 2021

\begin{abstract}
Acetylsalicylic acid (ASA) is widely used in the treatment and prevention of cardiovascular disorders. Our objective is to evaluate its possible protective role, not only in mortality but also in other aspects such as inflammation, symptomatic thrombosis, and intensive care unit (ICU) admission in hospitalized COVID-19 patients. We realized an observational retrospective cohort study of 20,641 patients with COVID-19 pneumonia collected and followed-up from Mar 1st, 2020 to May 1st, 2021, from the nationwide Spanish SEMI-COVID-19 Registry. Propensity score matching (PSM) was performed to determine whether treatment with ASA affected outcomes in COVID-19 patients. On hospital admission, 3291 (15.9\%) patients were receiving ASA. After PSM, 3291 patients exposed to ASA and 2885 not-exposed patients were analyzed. In-hospital mortality was higher in the ASA group (30.4 vs. $16.9 \%, p<0.001)$ in the global sample. After PSM, no differences were found between groups ( 30.4 vs. $30.3 \%, p=0.938)$. There were no differences in inflammation, symptomatic thrombosis, or ICU admission. In conclusion, ASA intake is not associated with in-hospital mortality or any other health outcome evaluated after applying PSM analysis in a real-world large sample of hospitalized COVID-19 patients.
\end{abstract}

Keywords Acetylsalicylic acid · Coronavirus · COVID-19 $\cdot$ Mortality

\section{Introduction}

The coronavirus disease 2019 (COVID-19), caused by the severe acute respiratory syndrome coronavirus 2 (SARS$\mathrm{CoV}-2$ ), produces severe respiratory symptoms such as bilateral pneumonia associated with high morbidity and

Francesc Formiga and Manuel Rubio-Rivas contributed equally as co-first authors of the study.

A complete list of the SEMI-COVID-19 Network members is provided in the Appendix.

Francesc Formiga

fformiga@bellvitgehospital.cat

Extended author information available on the last page of the article mortality, especially in patients of advanced age [1]. At this time, there is no known active treatment to fight this virus. Corticosteroids and biological immunosuppressants are being used to treat the inflammatory phase of the disease [1].

As severe COVID-19 infection is mainly a multisystem inflammatory process with an increased risk of hypercoagulability, the use of acetylsalicylic acid (ASA) can theoretically provide positive outcomes [2,3]. It inhibits platelet aggregation triggered by the release of arachidonic acid from platelet cells [4]. Thus, the possible mechanism of the protective effects of ASA may be related to its antithrombotic and anti-inflammatory effects and also to its possible immunomodulatory effects (antiviral activity against DNA and RNA viruses, including different human coronaviruses) [5].

The role of ASA in patients with COVID-19 is not studied in depth. A first meta-analysis, including three studies 
evaluating the association between ASA at admission use and mortality in COVID-19 hospitalized patients, suggested no association between the use of ASA and mortality in patients with COVID-19 [6]. Although patients on ASA tend to have more risk factors for severe COVID-19 infection (older age, high cardiovascular risk, pre-existing coronary artery disease, etc.), the low heterogeneity in this analysis despite differences in characteristics of the population of the included studies [7-9] likely suggests no protective effect of ASA among different groups of patients [6].

As ASA was widely used in the treatment and prevention in the real world in cardiovascular disorders, our study aimed to check its possible protective role, not only in mortality but in other aspects such as inflammation, symptomatic thrombosis, and intensive care unit (ICU) admission.

\section{Materials and methods}

\section{Study design, patient selection, and data collection}

The analysis was performed in the large cohort of consecutive patients included in the Spanish SEMI-COVID-19 Registry, created by the Spanish Society of Internal Medicine (SEMI). This is a multicenter, nationwide registry with over 150 hospitals registered so far. From March 1st, 2020 to May 1st, 2021, 21,962 hospitalized patients were included in the Registry. Methods of the study have been previously described [10]. In brief, all included patients were diagnosed by polymerase chain reaction (PCR) test or rapid antigenic test for SARS-CoV-2 taken from a nasopharyngeal sample, sputum, or bronchoalveolar lavage. The collection of data from each patient in terms of sociodemographic data, comorbidities, laboratory data, treatments, and outcomes was verified by the principal investigator of each center through the review of clinical records. All participating centers in the register received confirmation from the relevant Ethics Committees, including Bellvitge University Hospital (PR 128/20).

The inclusion criteria were all patients in the registry with a community (non-nosocomial) SARS-CoV-2 infection. We included all patients with valid information on whether or not they were taking ASA at the time of hospital admission. The patient sample was divided into 2 groups: patients with ASA admission intake and patients without.

The treatments received were in accordance with the medical guidelines available at the time of the pandemic [10]. In the absence of clinical evidence of any of the treatments at the initial time of the pandemic, their use was allowed off-label.

\section{Outcomes definition}

The primary outcome of the study was in-hospital mortality. Secondary outcomes were the development of symptomatic deep venous thrombosis (DVT) or pulmonary embolism (PE), the requirement of high-flow nasal cannula (HFNC), noninvasive mechanical ventilation (NIMV), invasive mechanical ventilation (IMV), ICU admission, and the combined variable of in-hospital mortality, the requirement of HFNC, NIMV, IMV, or ICU admission. Also, the development of inflammation in the high-risk category according to the categories previously defined by our group [10]. This was defined when the patient met 1 of the following criteria: lymphocyte count $<760 \times 106 / \mathrm{L}$, C-reactive protein $(\mathrm{CRP})>101.5 \mathrm{mg} / \mathrm{L}$, lactate dehydrogenase $(\mathrm{LDH})>394 \mathrm{U} / \mathrm{L}$, ferritin $>1359.9$ $\mathrm{mcg} / \mathrm{L}$, or D-dimer $>1580 \mathrm{ng} / \mathrm{mL}$.

\section{Statistical analysis}

Multiple imputations of missing data were performed. To minimize differences between groups and improve comparability, logistic-regression propensity score nearest neighbor matching (PSM) with replacement and caliper 0.2 was performed. The PSM included sociodemographic variables (age and sex), days from symptom onset to hospital admission, smoking behavior, body mass index (BMI), comorbidities as arterial hypertension, diabetes mellitus, dyslipidemia, chronic liver disease, severe chronic renal failure, chronic obstructive pulmonary disease (COPD), asthma, obstructive sleep apnea syndrome (OSAS), chronic heart failure, ischemic heart disease, cerebrovascular disease, cancer, dementia, degree of dependency, and Charlson index, tachypnea and laboratory variables on admission as $\mathrm{PaO} 2 / \mathrm{FiO} 2$, ferritin, lactate dehydrogenase (LDH), C-reactive protein (CRP), lymphocyte count, D-dimer, albumin, creatinine, platelets count, and hemoglobin), and treatments during admission (corticosteroids, tocilizumab, and remdesivir).

Categorical variables were expressed as absolute numbers and percentages. Continuous variables are expressed as mean plus standard deviation (SD) in the case of parametric distribution or median [IQR] in the case of non-parametric distribution. Differences among groups were assessed using the chi-square test for categorical variables and T-test or Mann-Whitney test as appropriate for continuous variables. $p$ values $<0.05$ indicated statistical significance.

Statistical analysis was performed by IBM SPSS Statistics for Windows, Version 26.0. Armonk, NY, USA: IBM Corp. 


\section{Results}

We included 20,641 patients in the study. There were 11,879 men (57\%). On hospital admission, 3291 (15.9\%) patients were detected as receiving ASA. In an attempt to differentiate the ASA effect from other effects, 3,291 exposed to ASA and 2885 not-exposed patients were matched.

\section{General baseline data between groups}

In the unadjusted analysis, patients on admission ASA were significantly older, predominantly male, and with fewer days between the start of symptoms at admission. Furthermore, patients on ASA were more frequently former smokers and had higher dependence, more comorbidities (arterial hypertension, dyslipidemia, diabetes mellitus, ischemic cardiopathy, cerebrovascular disease, peripheral arterial disease, dementia, chronic heart failure, severe chronic renal failure, cancer, COPD, and OSAS), and higher score in the Charlson Index. In contrast, the percentage of patients with atrial fibrillation and asthma was lower in patients with admission ASA use (Table 1).

The differences regarding days from onset to admission, degree of dependency, arterial hypertension, diabetes mellitus, cerebrovascular disease, dementia, and chronic heart failure disappeared after PSM analysis.

Regarding symptoms, ASA patients had a lower percentage of cough, arthromyalgias, ageusia, anosmia, sore throat, headache, fever, diarrhea, and abdominal pain with a similar percentage of dyspnea and vomiting. Heart rate was lower in ASA patients on admission, although in this group there was a higher percentage of patients with respiratory rate $>20 \mathrm{bpm}$ (Table 2). All symptoms and physical examination differences lost the statistical association after PSM.

\section{Laboratory tests between groups}

Concerning to the analytical values, the group with ASA admission use had lower $\mathrm{PaO} 2 / \mathrm{FiO} 2$, lymphocyte count, platelet figures, and albumin. In contrast, they presented with higher values of CRP, LDH, D-dimer, and creatinine. In the group without ASA, they presented higher values of ferritin and alanine transferase (ALT) (Table 3). Except for the Ddimer, all variables lost their association after PSM.

\section{Treatments during admission}

During admission (Table 4), patients with ASA use were treated less frequently with oral anticoagulants, tocilizumab, and remdesivir. In contrast, they were treated more often with prophylactic doses of low-molecular-weight heparin (LMWH) and corticosteroids. Only treatment with LMWH retained significance when applying PSM.

\section{Outcomes between groups (Fig. 1)}

In-hospital mortality was higher in the ASA group (30.4 vs. $16.9 \%, p<0.001)$. ASA patients presented also more frequently with high-risk-inflammatory categories $(80.1$ vs. $75.3 \%, p<0.001)$, the requirement of NIMV (6.7 vs. $5.7 \%, p=0.028)$, and the combined variable (38.1 vs. $27.4 \%, p<0.001)$. In contrast, ASA patients required less frequent IMV (6.5 vs. 7.7\%, $p=0.014)$ and ICU admission ( 8.6 vs. $9.9 \%, p=0.018$ ). There were no differences in the number of DVT ( 0.5 vs. $0.6 \%, p=0.851)$, PE (1.7 vs. $1.7 \%$, $p=0.851), \mathrm{DVT}+\mathrm{PE}(0.1$ vs. $0.2 \%, p=0.851)$, or HFNC use $(9.7$ vs. $9.5 \%, p=0.720)$. As shown in Table 5 , none of the outcomes assessed remained associated with admission ASA use after PSM. In-depth, in-hospital mortality was practically identical in the two groups after matching (30.4 vs. $30.3 \%, p=0.938)$, same as DVT (0.5 vs $0.8 \%$, $p=0.508), \operatorname{PE}(1.7$ vs. $1.4 \%, p=0.508), \mathrm{DVT}+\mathrm{PE}(0.1$ vs $0.2 \%, p=0.508$ ), high-risk inflammation category (80.1 vs. $79.1 \%, p=0.316)$, HFNC (9.7 vs. $9.1 \%, p=0.411)$, NIMV ( 6.7 vs. $6.3 \%, p=0.518)$, IMV (6.5 vs. $6.3 \%, p=0.750)$, ICU ( 8.6 vs. $8.2 \%, p=0.622)$, and the combined variable ( 38.1 vs. $38 \%, p=0.946)$.

\section{Risk factors for in-hospital mortality (Table 6)}

Despite PSM some variables were not correctly matched so we performed a logistic regression to really investigate the possible effectiveness of ASA. The factors that were related to higher in-hospital mortality were older age, male sex, higher degree of dependency, chronic heart failure, higher Charlson index, tachypnea on admission, higher CRP, LDH, and ferritin levels. Lower $\mathrm{PaO} 2 / \mathrm{FiO} 2$ and lymphocyte count levels were also associated with higher in-hospital mortality. The use of ASA was not related to in-hospital mortality.

\section{Discussion}

The main result of our study in a very large sample of patients (more than 20,000 patients) is the fact that there is no relationship between the intake of ASA at admission and the main outcome (in-hospital mortality), nor the secondary outcomes (inflammation, symptomatic thrombosis, and ICU admission) in Spanish hospitalized patients with COVID-19 infection after the correcting impact of PSM analysis.

ASA-related antiplatelet and anti-inflammatory effects could lead to better health outcomes in patients hospitalized for COVID-19 but our study failed to demonstrate that. 
Table 1 Patient characteristics before and after propensity score matching

\begin{tabular}{|c|c|c|c|c|c|c|c|c|}
\hline & \multicolumn{4}{|l|}{ All cohort } & \multicolumn{4}{|c|}{ Propensity Score Matched Cohort } \\
\hline & ASA & No ASA & $\%$ or mean difference & $p$ value & ASA & No ASA & $\begin{array}{l}\% \text { or mean } \\
\text { difference }\end{array}$ & $p$ value \\
\hline $\mathrm{N}$ & 3291 & 17,350 & & & 3291 & 2885 & & \\
\hline Age, median [IQR] & $77.6[70-85.2]$ & $66.9[54.4-78.4]$ & +10.7 & $<0.001$ & $77.6[70-85.2]$ & 79 [70.6-86.1] & -1.4 & 0.003 \\
\hline $\begin{array}{l}\text { Gender (males), } n \\
(\%)\end{array}$ & $2053(62.4)$ & $9826(56.6)$ & $+5.8 \%$ & $<0.001$ & $2053(62.4)$ & $1704(59.1)$ & $+3.3 \%$ & 0.008 \\
\hline $\begin{array}{l}\text { Race, } n(\%) \\
\text { Caucasian } \\
\text { Black } \\
\text { Hispanic } \\
\text { Asian } \\
\text { Others }\end{array}$ & $\begin{array}{l}3174(96.4) \\
10(0.3) \\
71(2.2) \\
7(0.2) \\
29(0.9)\end{array}$ & $\begin{array}{l}15,292(88.1) \\
105(0.6) \\
1629(9.4) \\
95(0.5) \\
229(1.3)\end{array}$ & $\begin{array}{l}+8.3 \% \\
-0.3 \% \\
-7.2 \% \\
-0.3 \% \\
-0.4 \%\end{array}$ & $<0.001$ & $\begin{array}{l}3174(96.4) \\
10(0.3) \\
71(2.2) \\
7(0.2) \\
29(0.9)\end{array}$ & $\begin{array}{l}2782(96.4) \\
9(0.3) \\
68(2.4) \\
7(0.2) \\
29(0.9)\end{array}$ & $\begin{array}{l}0 \\
0 \\
+0.2 \% \\
0 \\
0\end{array}$ & 0.859 \\
\hline $\begin{array}{l}\text { Days from onset to } \\
\text { admission, median } \\
\text { [IQR] }\end{array}$ & $5[3-8]$ & $7[4-9]$ & -2 & $<0.001$ & $5[3-8]$ & $5[3-8]$ & 0 & 0.647 \\
\hline BMI, median [IQR] & $28.6[25.4-32]$ & $28.5[25.3-32.1]$ & +0.1 & 0.793 & $28.6[25.4-32]$ & $28.5[25.3-32]$ & +0.1 & 0.653 \\
\hline $\begin{array}{l}\text { Smoking behavior, } \\
n(\%) \\
\text { Never smoker } \\
\text { Former smoker } \\
\text { Current smoker }\end{array}$ & $\begin{array}{l}1912(58.1) \\
1220(37.1) \\
159(4.8)\end{array}$ & $\begin{array}{l}12,460(71.8) \\
4055(23.4) \\
834(4.8)\end{array}$ & $\begin{array}{l}-13.7 \% \\
+13.7 \% \\
0\end{array}$ & $<0.001$ & $\begin{array}{l}1912(58.1) \\
1220(37.1) \\
159(4.8)\end{array}$ & $\begin{array}{l}1805(62.6) \\
925(32.1) \\
155(5.4)\end{array}$ & $\begin{array}{l}-4.5 \% \\
+5 \% \\
-0.6 \%\end{array}$ & $<0.001$ \\
\hline $\begin{array}{l}\text { Degree of depend- } \\
\text { ency, } n(\%) \\
\text { None or mild } \\
\text { Moderate } \\
\text { Severe }\end{array}$ & $\begin{array}{l}2340(71.1) \\
532(16.2) \\
419(12.7)\end{array}$ & $\begin{array}{l}14,847(85.6) \\
1419(8.2) \\
1084(6.2)\end{array}$ & $\begin{array}{l}-14.5 \% \\
+8 \% \\
+6.5 \%\end{array}$ & $<0.001$ & $\begin{array}{l}2340(71.1) \\
532(16.2) \\
419(12.7)\end{array}$ & $\begin{array}{l}2021(70.1) \\
468(16.2) \\
396(13.7)\end{array}$ & $\begin{array}{l}+1 \% \\
0 \\
-1 \%\end{array}$ & 0.498 \\
\hline $\begin{array}{l}\text { Arterial hyperten- } \\
\text { sion, } n(\%)\end{array}$ & $2599(79)$ & 8109 (46.7) & $+32.3 \%$ & $<0.001$ & $2599(79)$ & $2260(78.3)$ & $+0.7 \%$ & 0.542 \\
\hline Dyslipidemia, $n(\%)$ & $2140(65)$ & $6046(34.8)$ & $+30.2 \%$ & $<0.001$ & $2140(65)$ & $1790(62)$ & $+3 \%$ & 0.015 \\
\hline $\begin{array}{l}\text { Diabetes mellitus, } \\
n(\%)\end{array}$ & $1235(37.5)$ & $3000(17.3)$ & $+20.2 \%$ & $<0.001$ & $1235(37.5)$ & $1021(35.4)$ & $+2.1 \%$ & 0.082 \\
\hline $\begin{array}{l}\text { Atrial fibrillation, } \\
n(\%)\end{array}$ & $283(8.6)$ & 1941 (11.2) & $-2.6 \%$ & $<0.001$ & $283(8.6)$ & $322(11.2)$ & $-2.6 \%$ & 0.001 \\
\hline $\begin{array}{l}\text { Ischemic cardiopa- } \\
\text { thy, } n(\%)\end{array}$ & 953 (29) & $665(3.8)$ & $+25.2 \%$ & $<0.001$ & 953 (29) & $538(18.6)$ & $+10.4 \%$ & $<0.001$ \\
\hline $\begin{array}{l}\text { Cerebrovascular } \\
\text { disease, } n(\%)\end{array}$ & $662(20.1)$ & $847(4.9)$ & $+15.2 \%$ & $<0.001$ & $662(20.1)$ & $524(18.2)$ & $+1.9 \%$ & 0.052 \\
\hline $\begin{array}{l}\text { Peripheral arterial } \\
\text { disease, } n(\%)\end{array}$ & $402(12.2)$ & $471(2.7)$ & $+9.5 \%$ & $<0.001$ & $402(12.2)$ & $275(9.5)$ & $+2.7 \%$ & 0.001 \\
\hline Dementia, $n(\%)$ & $567(17.2)$ & $1,459(8.4)$ & $+8.8 \%$ & $<0.001$ & $567(17.2)$ & $521(18.1)$ & $-0.9 \%$ & 0.393 \\
\hline $\begin{array}{l}\text { Chronic heart fail- } \\
\text { ure, } n(\%)\end{array}$ & $434(13.2)$ & $946(5.5)$ & $+7.7 \%$ & $<0.001$ & $434(13.2)$ & 338 (11.7) & $+1.5 \%$ & 0.081 \\
\hline $\begin{array}{l}\text { Chronic liver dis- } \\
\text { ease, } n(\%)\end{array}$ & $124(3.8)$ & $578(3.3)$ & $+0.5 \%$ & 0.205 & $124(3.8)$ & $133(4.6)$ & $-0.8 \%$ & 0.098 \\
\hline $\begin{array}{l}\text { Severe chronic renal } \\
\text { failure, } n(\%)\end{array}$ & $390(11.9)$ & $816(4.7)$ & $+7.2 \%$ & $<0.001$ & $390(11.9)$ & $312(10.8)$ & $+1.1 \%$ & 0.201 \\
\hline Cancer, $n(\%)$ & $372(11.3)$ & $1566(9)$ & $+2.3 \%$ & $<0.001$ & $372(11.3)$ & $353(12.2)$ & $-0.9 \%$ & 0.256 \\
\hline COPD, $n(\%)$ & $371(11.3)$ & $1029(5.9)$ & $+5.4 \%$ & $<0.001$ & $371(11.3)$ & $317(11)$ & $+0.3 \%$ & 0.722 \\
\hline Asthma, $n(\%)$ & $195(5.9)$ & $1257(7.2)$ & $-1.3 \%$ & 0.007 & $195(5.9)$ & $188(6.5)$ & $-0.6 \%$ & 0.336 \\
\hline OSAS, $n(\%)$ & $271(8.2)$ & $966(5.6)$ & $+2.6 \%$ & $<0.001$ & $271(8.2)$ & $222(7.7)$ & $+0.5 \%$ & 0.435 \\
\hline $\begin{array}{l}\text { Charlson index } \\
\text { median [IQR] }\end{array}$ & $2[1-3]$ & $0[0-2]$ & +2 & $<0.001$ & $2[1-3]$ & $1[1-3]$ & +1 & $<0.001$ \\
\hline
\end{tabular}

ASA Acetylsalicylic acid, $B M I$ body mass index, $I Q R$ interquartile range, $C O P D$ chronic obstructive pulmonary disease, $O S A S$ obstructive sleep apnea syndrome, Severe chronic renal failure: Creatinine $>300 \mathrm{mg} / \mathrm{dl}$ or dialysis 
Table 2 Symptoms and physical examination upon admission between groups before and after propensity score matching

\begin{tabular}{|c|c|c|c|c|c|c|c|c|}
\hline & \multicolumn{4}{|l|}{ All cohort } & \multicolumn{4}{|c|}{ Propensity score matched cohort } \\
\hline & ASA & No ASA & $\begin{array}{l}\% \text { or mean } \\
\text { difference }\end{array}$ & $p$ value & ASA & No ASA & $\begin{array}{l}\% \text { or mean } \\
\text { difference }\end{array}$ & $p$ value \\
\hline Cough, $n(\%)$ & $2148(65.3)$ & $12,534(72.2)$ & $-6.9 \%$ & $<0.001$ & $2148(65.3)$ & $1864(64.6)$ & $+0.7 \%$ & 0.588 \\
\hline Arthromyalgias, $n(\%)$ & $736(22.4)$ & $5375(31)$ & $-8.6 \%$ & $<0.001$ & $736(22.4)$ & $637(22.1)$ & $+0.3 \%$ & 0.789 \\
\hline Ageusia, $n(\%)$ & $184(5.6)$ & $1730(10)$ & $-4.4 \%$ & $<0.001$ & $184(5.6)$ & $164(5.7)$ & $-0.1 \%$ & 0.874 \\
\hline Anosmia, $n(\%)$ & $151(4.6)$ & $1535(8.8)$ & $-4.2 \%$ & $<0.001$ & $151(4.6)$ & $146(5.1)$ & $-0.5 \%$ & 0.387 \\
\hline Sore throat, $n(\%)$ & $230(7)$ & $1683(9.7)$ & $-2.7 \%$ & $<0.001$ & $230(7)$ & $209(7.2)$ & $-0.2 \%$ & 0.697 \\
\hline Headache, $n(\%)$ & $230(7)$ & $2264(13)$ & $-6 \%$ & $<0.001$ & $230(7)$ & $196(6.8)$ & $+0.2 \%$ & 0.763 \\
\hline Fever, $n(\%)$ & $2487(75.6)$ & $14,261(82.2)$ & $-6.6 \%$ & $<0.001$ & $2487(75.6)$ & $2168(75.1)$ & $+0.5 \%$ & 0.701 \\
\hline Dyspnea, n (\%) & $1928(58.6)$ & $10,200(58.8)$ & $-0.2 \%$ & 0.826 & $1928(58.6)$ & $1685(58.4)$ & $+0.2 \%$ & 0.887 \\
\hline Diarrhea, $n(\%)$ & $692(21)$ & $4257(24.5)$ & $-3.5 \%$ & $<0.001$ & $692(21)$ & $588(20.4)$ & $+0.6 \%$ & 0.532 \\
\hline Vomiting, $n(\%)$ & $246(7.5)$ & $1367(7.9)$ & $-0.4 \%$ & 0.429 & $246(7.5)$ & $210(7.3)$ & $+0.2 \%$ & 0.769 \\
\hline Abdominal pain, $n(\%)$ & $179(5.4)$ & $1128(6.5)$ & $-1.1 \%$ & 0.022 & $179(5.4)$ & $188(6.5)$ & $-1.1 \%$ & 0.074 \\
\hline Heart rate, bpm median [IQR] & 83 [73-95] & $88[77-100]$ & -5 & $<0.001$ & 83 [73-95] & 84 [74-95] & -1 & 0.077 \\
\hline Respiratory rate $>20 \mathrm{bpm}, n(\%)$ & $1142(34.7)$ & $5429(31.3)$ & $+3.4 \%$ & $<0.001$ & $1142(34.7)$ & 1037 (35.9) & $-1.2 \%$ & 0.307 \\
\hline
\end{tabular}

ASA Acetylsalicylic acid, IQR interquartile range

Table 3 Laboratory tests upon admission between groups before and after propensity score matching

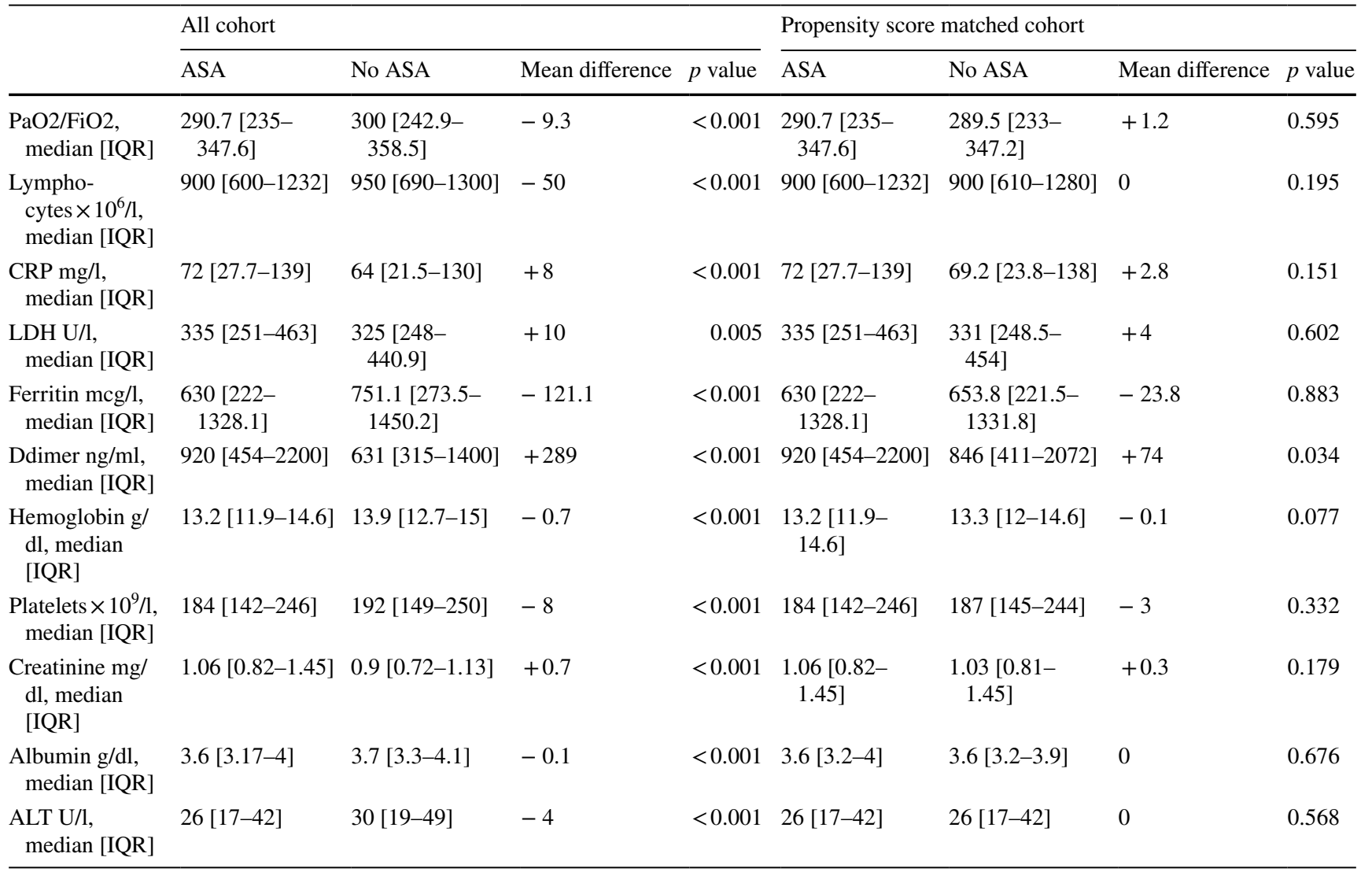

$A S A$ Acetylsalicylic acid, $A L T$ alanine transferase. $C R P$ C-reactive protein, $L D H$ lactate dehydrogenase, $I Q R$ interquartile range 
Table 4 Treatments during admission between groups before and after propensity score matching

\begin{tabular}{|c|c|c|c|c|c|c|c|c|}
\hline & \multicolumn{4}{|l|}{ All cohort } & \multicolumn{4}{|c|}{ Propensity score matched cohort } \\
\hline & ASA & No ASA & $\%$ difference & $p$ value & ASA & No ASA & \%difference & $p$ value \\
\hline $\begin{array}{l}\text { Oral anticoagulants, } n(\%) \\
\text { Anti vitamin k } \\
\text { DOAC }\end{array}$ & $\begin{array}{l}33(1) \\
46(1.4)\end{array}$ & $\begin{array}{l}293(1.7) \\
402(2.3)\end{array}$ & $\begin{array}{l}-0.7 \% \\
-0.9 \%\end{array}$ & $<0.001$ & $\begin{array}{l}33(1) \\
46(1.4)\end{array}$ & $\begin{array}{l}46(1.6) \\
52(1.8)\end{array}$ & $\begin{array}{l}-0.6 \% \\
-0.4 \%\end{array}$ & 0.051 \\
\hline $\begin{array}{l}\text { LMWH, } n(\%) \\
\text { None } \\
\text { Prophylactic doses } \\
\text { Intermediate doses } \\
\text { Full doses }\end{array}$ & $\begin{array}{l}371(12.3) \\
2208(67.1) \\
315(9.6) \\
364(11.1)\end{array}$ & $\begin{array}{l}2737(16.3) \\
10,991(63.3) \\
1493(8.6) \\
2032(11.7)\end{array}$ & $\begin{array}{l}-4 \% \\
+3.8 \% \\
+1 \% \\
-0.6 \%\end{array}$ & $<0.001$ & $\begin{array}{l}404(12.3) \\
2208(67.1) \\
364(11.1) \\
315(9.6)\end{array}$ & $\begin{array}{l}406(14.1) \\
1812(62.8) \\
403(14) \\
264(9.2)\end{array}$ & $\begin{array}{l}-1.8 \% \\
+4.3 \% \\
-2.9 \% \\
+0.4 \%\end{array}$ & $<0.001$ \\
\hline Steroids, $n(\%)$ & $1663(50.9)$ & $7817(45.3)$ & $+5.6 \%$ & $<0.001$ & $1663(50.5)$ & 1439 (49.9) & $+0.6 \%$ & 0.609 \\
\hline Tocilizumab, $n(\%)$ & $272(8.3)$ & $1748(10.1)$ & $-1.8 \%$ & 0.001 & $272(8.3)$ & $236(8.2)$ & $+0.1 \%$ & 0.904 \\
\hline Remdesivir, $n(\%)$ & $126(3.8)$ & $823(4.7)$ & $-0.9 \%$ & 0.022 & $126(3.8)$ & $106(3.7)$ & $+0.1 \%$ & 0.750 \\
\hline
\end{tabular}

ASA Acetylsalicylic acid, DOACs direct oral anticoagulants, $L M W \mathrm{H}$ low-mollecular-weight heparin

Fig. 1 Outcomes between groups after propensity score matching. ASA Acetylsalicylic acid, HFNC high Flow nasal cannula, NIMV non-invasive mechanical ventilation, $I M V$ invasive mechanical ventilation, $I C U$ intensive care unit, $D V T$ deep venous thrombosis, $P E$ pulmonary embolism

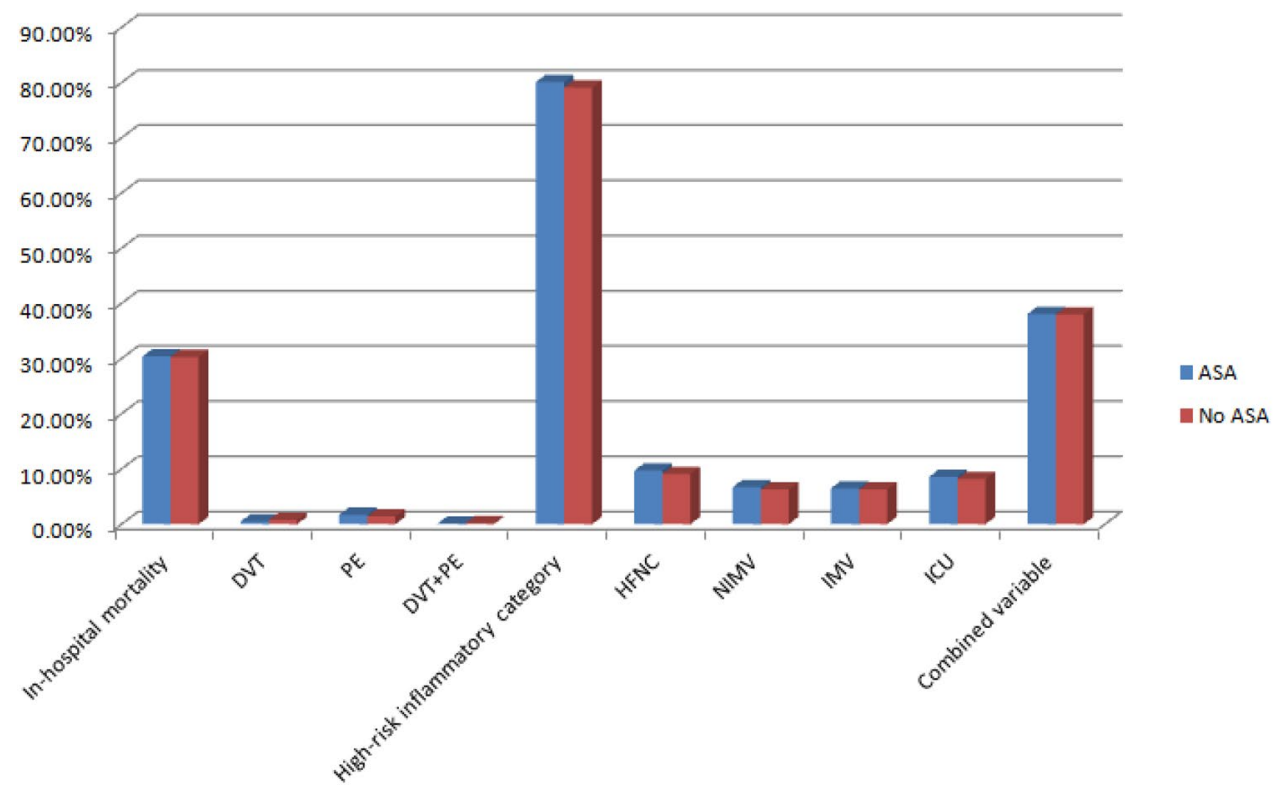

When evaluating the data globally, the percentage of inhospital mortality was much higher in the ASA group (30.4 vs. $16.9 \%, p<0.001)$. The most obvious hypothesis is that occurs because the ASA patients were older and with more comorbidities. When applied PSM, we found identical rates of $30 \%$ of in-hospital mortality irrespective of whether or not they had previously taken ASA.

ASA is inexpensive, widely available, and with clear indications of prescription and a well-known risk profile. Of all the patients admitted for COVID-19 in Spain, 15.9\% were under ASA before hospital admission. The percentage of patients admitted for COVID-19 who were under ASA is slightly lower than the $19.2 \%$ reported in a recent meta-analysis [6] and the 24\% reported in a Veterans Health Administration study in patients with COVID-19 infection [11]. Our results confirm, as previously reported in COVID-19 patients, that ASA users tend to have more risk factors for severe COVID-19 infection (older age, diabetes mellitus, ischemic cardiopathy, etc.,)[6].

In-hospital mortality among ASA users in the metaanalysis by Salah et al.[6] was 22.6 vs. $18.3 \%$ among nonASA users ( $\mathrm{RR}=1.12,95 \% \mathrm{CI} 0.84-1.50)$. It is important to take into account the few patients included in the 3 studies, and none of them assessing a European population. So, in the study by Alamdari et al. [7] they evaluated 459 patients (53 under ASA) from Iran, Chow et al. [9] included 419 USA patients (98 under ASA), and Yuan et al. [8] 183 Chinese patients, all with coronary artery disease (52 under ASA). We evaluated higher figures of patients (20,641 patients) of whom 3291 were treated with ASA before hospital admission. Sahai et al. [12] concluded in 248 USA-matched patients with COVID-19 that ASA 
Table 5 Outcomes between groups before and after propensity score matching

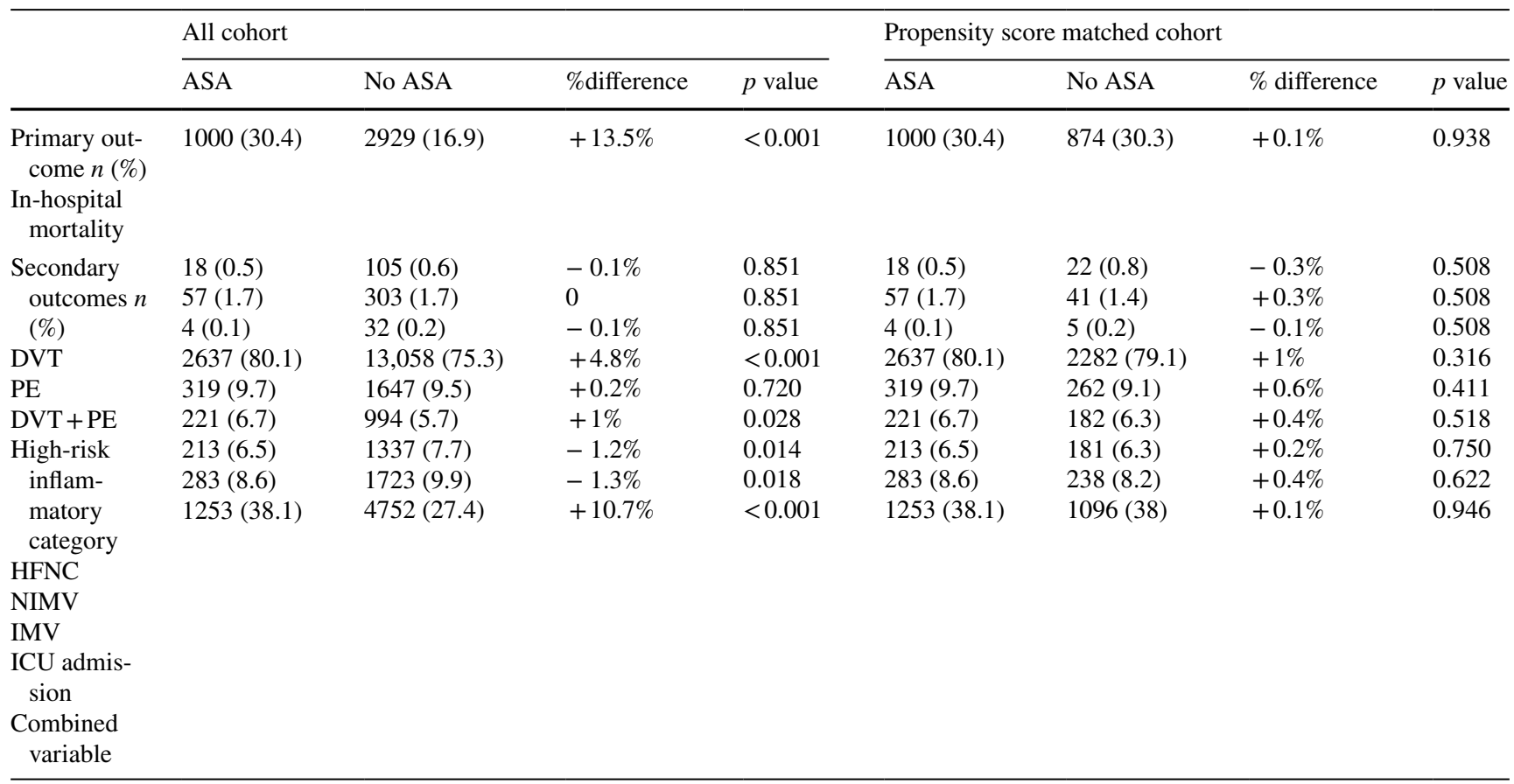

ASA Acetylsalicylic acid, HFNC High Flow nasal cannula, NIMV non-invasive mechanical ventilation, IMV invasive mechanical ventilation, $I C U$ intensive care unit, DVT Deep venous thrombosis, PE Pulmonary embolism

had no overall mortality benefit in a retrospective observational study.

A recent study from Iran assessing 991 patients (336 with prior ASA intake or having started ASA on the first day of hospital admission) demonstrated a significant independent association between ASA and lower in-hospital mortality ( $R R=0.75,95 \%$ CI 0.56-0.99) [13]. Although the study was not carried out with PSM correction, it is worth highlighting the results taking into account that patients with ASA were older and had more comorbidities. Finally, Chow et al. [9] reported that ASA use was independently associated with decreased risk of MV and ICU admission. Neither Yuan et al. [8] nor our results after PSM could confirm this association.

In a different scenario from our study, ASA has shown good health outcomes. Liu et al. [14] enrolled 24 pairs of patients (after PSM) and reported that among adults (with arterial hypertension and cardiovascular diseases) infected with SARS-CoV-2, low-dose ASA (100 mg/day) was associated with a lower risk of mortality compared with non-ASA users. Among more than 30,000 COVID-19 positive USA Veterans, prior ASA intake was associated with a statistically and clinically significant decrease in overall mortality at 14 -days $(\mathrm{OR}=0.38,95 \% \mathrm{CI} 0.32-0.46)$ and 30-days $(\mathrm{OR}=0.38,95 \%$ CI $0.33-0.45)$ [13]. One possible interpretation of these results is that ASA is beneficial due to its possible antithrombotic, anti-inflammatory, and immunomodulation effects in community cases of COVID-19, but no longer when patients have a severe condition requiring hospital admission. A USA study [15] evaluating 1,956 patients according to the antiplatelet therapy prior to and during admission found lower in-hospital mortality in the group with ASA during admission after applying PSM (HR $=0.52,95 \%$ CI 0.34-0.81). A recent meta-analysis [16] reported that ASA intake (prior or initiated during hospitalization) was independently associated with lower mortality in patients with COVID-19. When analyzing the results of the study, it is important to take into account the possible favorable effects of other important cardiovascular drug classes, especially renin-angiotensin-aldosterone system inhibitors and statins $[17,18]$.

The main strength of the study is a large number of patients evaluated, which makes it the largest study on the subject to our knowledge. Secondly, its multicentre hospital nature spans a diverse geographic range, from an integrated longitudinal database.

This study has several limitations. First, it was a retrospective study. Second, the intake of ASA before admission was collected and confirmed but neither the dose nor the reason for its prescription was recorded. Third, as this study was focused on in-patients, it was difficult to reflect the effect of ASA in an outpatient setting. Fourth, our sample is mostly Caucasian with a low representation of other ethnic groups. Fifth, we did not record the presence of other medications that are associated with hypercoagulabilities, such as oral contraceptives and hormone replacement. Sixth, we not explore if there was 
Table 6 Risk factors for in-hospital mortality in the matched-cohort

\begin{tabular}{|c|c|c|c|c|}
\hline & \multicolumn{2}{|l|}{ Univariate analysis } & \multicolumn{2}{|c|}{ Multivariate analysis } \\
\hline & OR $(95 \%$ CI $)$ & $p$ value & OR (95\% CI) & $p$ value \\
\hline Age & $1.06(1.05-1.07)$ & $<0.001$ & $1.19(1.02-1.38)$ & 0.030 \\
\hline Gender (female) & $0.85(0.76-0.95)$ & 0.005 & $0.73(0.63-0.83)$ & $<0.001$ \\
\hline BMI & $1.02(1.01-1.03)$ & 0.003 & NS & \\
\hline $\begin{array}{l}\text { Smoking behavior } \\
\text { Never (ref.) } \\
\text { Former smoker } \\
\text { Current smoker }\end{array}$ & $\begin{array}{l}1 \\
1.19(1.07-1.34) \\
0.83(0.64-1.08)\end{array}$ & $\begin{array}{l}0.002 \\
0.161\end{array}$ & NS & \\
\hline $\begin{array}{l}\text { Degree of dependency } \\
\text { Mild (ref.) } \\
\text { Moderate } \\
\text { Severe }\end{array}$ & $\begin{array}{l}1 \\
2.47(2.14-2.85) \\
3.03(2.60-3.53)\end{array}$ & $\begin{array}{l}<0.001 \\
<0.001\end{array}$ & $\begin{array}{l}1 \\
1.54(1.29-1.84) \\
2(1.65-2.42)\end{array}$ & $\begin{array}{l}<0.001 \\
<0.001\end{array}$ \\
\hline Arterial hypertension & $1.45(1.26-1.67)$ & $<0.001$ & NS & \\
\hline Dyslipidemia & $1.04(0.93-1.16)$ & 0.545 & & \\
\hline Diabetes mellitus & $1.17(1.04-1.30)$ & 0.008 & NS & \\
\hline Ischaemic cardiopathy & $1.36(1.20-1.54)$ & $<0.001$ & NS & \\
\hline Chronic heart failure & $2.14(1.83-2.49)$ & $<0.001$ & $1.21(1.01-1.47)$ & 0.049 \\
\hline Atrial fibrillation & $1.96(1.66-2.33)$ & $<0.001$ & NS & \\
\hline Cerebrovascular disease & $1.41(1.23-1.61)$ & $<0.001$ & NS & \\
\hline Peripheral arterial disease & $1.53(1.30-1.81)$ & $<0.001$ & NS & \\
\hline Dementia & $2.26(1.97-2.58)$ & $<0.001$ & NS & \\
\hline COPD & $1.46(1.24-1.72)$ & $<0.001$ & NS & \\
\hline Chronic liver disease & $1.04(0.79-1.36)$ & 0.780 & & \\
\hline Severe chronic renal failure & $1.85(1.58-2.17)$ & $<0.001$ & NS & \\
\hline Charlson index & $1.19(1.16-1.22)$ & $<0.001$ & $1.12(1.08-1.16)$ & $<0.001$ \\
\hline Respiratory rate $>20 \mathrm{rpm}$ & $3.65(3.26-4.10)$ & $<0.001$ & $2.49(2.19-2.84)$ & $<0.001$ \\
\hline $\mathrm{PaO} 2 / \mathrm{FiO} 2$ & $0.99(0.99-0.99)$ & $<0.001$ & $0.99(0.99-0.99)$ & $<0.001$ \\
\hline Lymphocyte count $\times 10^{6} / \mathrm{L}$ & $0.99(0.99-0.99)$ & $<0.001$ & $0.99(0.99-0.99)$ & $<0.001$ \\
\hline CRP (mg/L) & $1.01(1.01-1,01)$ & $<0.001$ & $1.01(1.01-1.01)$ & $<0.001$ \\
\hline LDH (U/L) & $1.01(1.01-1,01)$ & $<0.001$ & $1.01(1.01-1.01)$ & $<0.001$ \\
\hline Ferritin $(\mathrm{mcg} / \mathrm{L})$ & $1.01(1.01-1,01)$ & $<0.001$ & $1.01(1.01-1.01)$ & 0.001 \\
\hline D-dimer (ng/mL) & $1.01(1.01-1.01)$ & $<0.001$ & NS & \\
\hline $\begin{array}{l}\text { LMWH } \\
\text { None (ref.) } \\
\text { Prophylactic doses } \\
\text { Intermediate doses } \\
\text { Full doses }\end{array}$ & $\begin{array}{l}1 \\
0.48(0.41-0.56) \\
0.57(0.46-0.72) \\
0.79(0.65-0.97)\end{array}$ & $\begin{array}{l}<0.001<0.001 \\
0.025\end{array}$ & $\begin{array}{l}1 \\
0.44(0.37-0.54) \\
0.41(0.31-0.53) \\
0.52(0.41-0.67)\end{array}$ & $\begin{array}{l}<0.001 \\
<0.001 \\
<0.001\end{array}$ \\
\hline Steroids & $1.46(1.31-1.63)$ & $<0.001$ & $1.22(1.07-1.4)$ & 0.003 \\
\hline Tocilizumab & $1.29(1.06-1.56)$ & 0.009 & $1.71(1.36-2.16)$ & $<0.001$ \\
\hline ASA & $1.01(0.90-1.12)$ & 0.938 & $1.05(0.92-1.19)$ & 0.476 \\
\hline
\end{tabular}

$B M I$ body mass index, $N S$ not significant, $C O P D$ chronic obstructive pulmonary disease, $C R P$ C-reactive protein, $L D H$ lactate dehydrogenase, $L M W H$ low-molecular-weight heparin, $A S A$ acetylsalicylic acid any difference in bleeding events Seventh, among the two groups, in-hospital mortality compared to patients who have undergone the same treatments for COVID was not evaluated. Finally, it is always concerning which variables to include in a PSM model. Including variables that are related to the exposure but not the outcome will decrease the precision of the estimated exposure effect without decreasing bias [20]. In our study, we have included a wide variety of comorbidities, some of them strongly related to ASA use but not so clearly related to the outcomes we describe. This could result in a loss of precision and, therefore, a limitation of the study.

\section{Conclusions}

To date, no consensus guidelines are available regarding ASA use in COVID-19, reflecting a paucity of data in this regard. Awaiting results from powered and randomized studies [19], 
our results, from a real-world large sample of hospitalized COVID-19 patients, provides information along with the idea that ASA intake at admission is not associated with lower inhospital mortality or any other health outcome evaluated after applying PSM analysis.

\section{Appendix}

\section{List of the SEMI-COVID-19 Network members}

Coordinator of the SEMI-COVID-19 Registry: José Manuel Casas Rojo.

SEMI-COVID-19 Scientific Committee Members: José Manuel Casas Rojo, José Manuel Ramos Rincón, Carlos Lumbreras Bermejo, Jesús Millán Núñez-Cortés, Juan Miguel Antón Santos, Ricardo Gómez Huelgas.

\section{Members of the SEMI-COVID-19 Group}

\section{H. Univ. de Bellvitge. L'Hospitalet de Llobregat (Barcelona)}

Xavier Corbella, Francesc Formiga, Narcís Homs, Abelardo Montero, Jose María Mora-Luján, Manuel Rubio-Rivas.

\section{H. U. 12 de Octubre. Madrid}

Paloma Agudo de Blas, Coral Arévalo Cañas, Blanca Ayuso, José Bascuñana Morejón, Samara Campos Escudero, María Carnevali Frías, Santiago Cossio Tejido, Borja de Miguel Campo, Carmen Díaz Pedroche, Raquel Diaz Simon, Ana García Reyne, Laura Ibarra Veganzones, Lucia Jorge Huerta, Antonio Lalueza Blanco, Jaime Laureiro Gonzalo, Jaime Lora-Tamayo, Carlos Lumbreras Bermejo, Guillermo Maestro de la Calle, Rodrigo Miranda Godoy, Barbara Otero Perpiña, Diana Paredes Ruiz, Marcos Sánchez Fernández, Javier Tejada Montes.

\section{H. Costa del Sol. Marbella (Málaga)}

Victoria Augustín Bandera, Javier García Alegría, Nicolás Jiménez-García, Jairo Luque del Pino, María Dolores Martín Escalante, Francisco Navarro Romero, Victoria Nuñez Rodriguez, Julián Olalla Sierra.

\section{H. U. Gregorio Marañon. Madrid}

Laura Abarca Casas, Álvaro Alejandre de Oña, Rubén Alonso Beato, Leyre Alonso Gonzalo, Jaime Alonso Muñoz, Crhistian Mario Amodeo Oblitas, Cristina Ausín García, Marta Bacete Cebrián, Jesús Baltasar Corral, Maria Barrientos Guerrero, Alejandro D. Bendala Estrada, María Calderón
Moreno, Paula Carrascosa Fernández, Raquel Carrillo, Sabela Castañeda Pérez, Eva Cervilla Muñoz, Agustín Diego Chacón Moreno, Maria Carmen Cuenca Carvajal, Sergio de Santos, Andrés Enríquez Gómez, Eduardo Fernández Carracedo, María Mercedes Ferreiro-Mazón Jenaro, Francisco Galeano Valle, Alejandra Garcia, Irene Garcia FernandezBravo, María Eugenia García Leoni, María Gómez Antúnez, Candela González San Narciso, Anthony Alexander Gurjian, Lorena Jiménez Ibáñez, Cristina Lavilla Olleros, Cristina Llamazares Mendo, Sara Luis García, Víctor Mato Jimeno, Clara Millán Nohales, Jesús Millán Núñez-Cortés, Sergio Moragón Ledesma, Antonio Muiño Míguez, Cecilia Muñoz Delgado, Lucía Ordieres Ortega, Susana Pardo Sánchez, Alejandro Parra Virto, María Teresa Pérez Sanz, Blanca Pinilla Llorente, Sandra Piqueras Ruiz, Guillermo Soria Fernández-Llamazares, María Toledano Macías, Neera Toledo Samaniego, Ana Torres do Rego, Maria Victoria Villalba Garcia, Gracia Villarreal, María Zurita Etayo.

\section{H. de Cabueñes. Gijón (Asturias)}

Ana María Álvarez Suárez, Carlos Delgado Vergés, Rosa Fernandez-Madera Martínez, Eva $\mathrm{M}^{\mathrm{a}}$ Fonseca Aizpuru, Alejandro Gómez Carrasco, Cristina Helguera Amezua, Juan Francisco López Caleya, Diego López Martínez, María del Mar Martínez López, Aleida Martínez Zapico, Carmen Olabuenaga Iscar, Lucía Pérez Casado, María Luisa Taboada Martínez, Lara María Tamargo Chamorro.

\section{H. Reg. Univ. de Málaga}

Ma Mar Ayala-Gutiérrez, Rosa Bernal López, José Bueno Fonseca, Verónica Andrea Buonaiuto, Luis Francisco Caballero Martínez, Lidia Cobos Palacios, Clara Costo Muriel, Francis de Windt, Ana Teresa Fernandez-Truchaud Christophel, Paula García Ocaña, Ricardo Gómez Huelgas, Javier Gorospe García, José Antonio Hurtado Oliver, Sergio Jansen-Chaparro, Maria Dolores López-Carmona, Pablo López Quirantes, Almudena López Sampalo, Elizabeth Lorenzo-Hernández, Juan José Mancebo Sevilla, Jesica Martín Carmona, Luis Miguel Pérez-Belmonte, Iván Pérez de Pedro, Araceli Pineda-Cantero, Carlos Romero Gómez, Michele Ricci, Jaime Sanz Cánovas.

\section{H. U. La Paz. Madrid}

Jorge Álvarez Troncoso, Francisco Arnalich Fernández, Francisco Blanco Quintana, Carmen Busca Arenzana, Sergio Carrasco Molina, Aranzazu Castellano Candalija, Germán Daroca Bengoa, Alejandro de Gea Grela, Alicia de Lorenzo 
Hernández, Alejandro Díez Vidal, Carmen Fernández Capitán, Maria Francisca García Iglesias, Borja González Muñoz, Carmen Rosario Herrero Gil, Juan María Herrero Martínez, Víctor Hontañón, Maria Jesús Jaras Hernández, Carlos Lahoz, Cristina Marcelo Calvo, Juan Carlos Martín Gutiérrez, Monica Martinez Prieto, Elena Martínez Robles, Araceli Menéndez Saldaña, Alberto Moreno Fernández, Jose Maria Mostaza Prieto, Ana Noblejas Mozo, Carlos Manuel Oñoro López, Esmeralda Palmier Peláez, Marina Palomar Pampyn, Maria Angustias Quesada Simón, Juan Carlos Ramos Ramos, Luis Ramos Ruperto, Aquilino Sánchez Purificación, Teresa Sancho Bueso, Raquel Sorriguieta Torre, Clara Itziar Soto Abanedes, Yeray Untoria Tabares, Marta Varas Mayoral, Julia Vásquez Manau.

\section{H. Royo Villanova. Zaragoza}

Nicolás Alcalá Rivera, Anxela Crestelo Vieitez, Esther del Corral Beamonte, Jesús Díez Manglano, Isabel Fiteni Mera, Maria del Mar Garcia Andreu, Martin Gericó Aseguinolaza, Cristina Gallego Lezaun, Claudia Josa Laorden, Raul Martínez Murgui, Marta Teresa Matía Sanz.

\section{H. Clínico de Santiago de Compostela (A Coruña)}

Maria del Carmen Beceiro Abad, Maria Aurora Freire Romero, Sonia Molinos Castro, Emilio Manuel Paez Guillan, María Pazo Nuñez, Paula Maria Pesqueira Fontan.

\section{H. Clínico San Carlos. Madrid}

Inés Armenteros Yeguas, Javier Azaña Gómez, Julia Barrado Cuchillo, Irene Burruezo López, Noemí Cabello Clotet, Alberto E. Calvo Elías, Elpidio Calvo Manuel, Carmen María Cano de Luque, Cynthia Chocron Benbunan, Laura Dans Vilan, Claudia Dorta Hernández, Ester Emilia Dubon Peralta, Vicente Estrada Pérez, Santiago FernandezCastelao, Marcos Oliver Fragiel Saavedra, José Luis García Klepzig, Maria del Rosario Iguarán Bermúdez, Esther Jaén Ferrer, Alejandro Maceín Rodríguez, Alejandro Marcelles de Pedro, Rubén Ángel Martín Sánchez, Manuel Méndez Bailón, Sara Miguel Álvarez, Maria José Nuñez Orantos, Carolina Olmos Mata, Eva Orviz García, David Oteo Mata, Cristina Outon González, Juncal Perez-Somarriba, Pablo Pérez Mateos, Maria Esther Ramos Muñoz, Xabier Rivas Regaira, Laura $M^{a}$ Rodríguez Gallardo, Iñigo Sagastagoitia Fornie, Alejandro Salinas Botrán, Miguel Suárez Robles, Maddalena Elena Urbano, Andrea María Vellisca González, Miguel Villar Martínez.

\section{H. Universitario Dr. Peset. Valencia}

Juan Alberto Aguilera Ayllón, Arturo Artero, María del Mar Carmona Martín, María José Fabiá Valls, Maria de Mar Fernández Garcés, Ana Belén Gómez Belda, Ian López Cruz, Manuel Madrazo López, Elisabeth Mateo Sanchis, Jaume Micó Gandia, Laura Piles Roger, Adela Maria Pina Belmonte, Alba Viana García.

\section{H. U. Puerta de Hierro. Madrid}

María Álvarez Bello, Ane Andrés Eisenhofer, Ana Arias Milla, Isolina Baños Pérez, Laura Benítez Gutiérrez, Javier Bilbao Garay, Silvia Blanco Alonso, Jorge Calderón Parra, Alejandro Callejas Díaz, José María Camino Salvador, $\mathrm{M}^{\mathrm{a}}$ Cruz Carreño Hernández, Valentín Cuervas-Mons Martínez, Sara de la Fuente Moral, Miguel del Pino Jimenez, Alberto Díaz de Santiago, Itziar Diego Yagüe, Ignacio Donate Velasco, Ana María Duca, Pedro Durán del Campo, Gabriela Escudero López, Esther Expósito Palomo, Ana Fernández Cruz, Esther Fiz Benito, Andrea Fraile López, Amy Galán Gómez, Sonia García Prieto, Claudia García RodríguezMaimón, Miguel Ángel García Viejo, Javier Gómez Irusta, Edith Vanessa Gutiérrez Abreu, Isabel Gutiérrez Martín, Ángela Gutiérrez Rojas, Andrea Gutiérrez Villanueva, Jesús Herráiz Jiménez, Pedro Laguna del Estal, $\mathrm{M}^{\mathrm{a}}$ Carmen Máinez Sáiz, Cristina Martín Martín, María Martínez Urbistondo, Fernando Martínez Vera, Susana Mellor Pita, Patricia Mills Sánchez, Esther Montero Hernández, Alberto Mora Vargas, Cristina Moreno López, Alfonso Ángel-Moreno Maroto, Victor Moreno-Torres, Concha, Ignacio Morrás De La Torre, Elena Múñez Rubio, Rosa Muñoz de Benito, Ana Muñoz Gómez, Alejandro Muñoz Serrano, Jose María Palau Fayós, Lina Marcela Parra Ramírez, Ilduara Pintos Pascual, Arturo José Ramos Martín-Vegue, Antonio Ramos Martínez, Isabel Redondo Cánovas del Castillo, Alberto Roldán Montaud, Lucía Romero Imaz, Yolanda Romero Pizarro, Enrique Sánchez Chica, David Sánchez Órtiz, Mónica Sánchez Santiuste, Patricia Serrano de la Fuente, Pablo Tutor de Ureta, Ángela Valencia Alijo, Mercedes Valentín-Pastrana Aguilar, Juan Antonio Vargas Núñez, Jose Manuel Vázquez Comendador, Gema Vázquez Contreras, Carmen Vizoso Gálvez.

\section{H. U. Reina Sofía. Córdoba}

Antonio Pablo Arenas de Larriva, Pilar Calero Espinal, Javier Delgado Lista, Francisco Fuentes-Jiménez, María del Carmen Guerrero Martínez, María Jesús Gómez Vázquez, Jose Jiménez Torres, Laura Limia Pérez, José 
López-Miranda, Laura Martín Piedra, Marta Millán Orge, Javier Pascual Vinagre, Pablo Pérez-Martinez, María Elena Revelles Vílchez, Angela Rodrigo Martínez, Juan Luis Romero Cabrera, José David Torres-Peña.

\section{H. U. de Badajoz}

Rafael Aragon Lara, Inmaculada Cimadevilla Fernandez, Juan Carlos Cira García, Gema Maria García García, Julia Gonzalez Granados, Beatriz Guerrero Sánchez, Francisco Javier Monreal Periáñez, Maria Josefa Pascual Perez.

\section{H. Moisès Broggi. Sant Joan Despí (Barcelona)}

Judit Aranda Lobo, Lucía Feria Casanovas, Jose Loureiro Amigo, Miguel Martín Fernández, Isabel Oriol Bermúdez, Melani Pestaña Fernández, Nicolas Rhyman, Nuria Vázquez Piqueras.

\section{H. U. Río Hortega. Valladolid}

Irene Arroyo Jiménez, Marina Cazorla González, Marta Cobos-Siles, Luis Corral-Gudino, Pablo Cubero-Morais, María González Fernández, José Pablo Miramontes González, Marina Prieto Dehesa, Pablo Sanz Espinosa.

\section{H. U. S. Juan de Alicante (Alicante)}

Marisa Asensio Tomás, David Balaz, David Bonet Tur, Ruth Cañizares Navarro, Paloma Chazarra Pérez, Jesús Corbacho Redondo, Eliana Damonte White, María Escamilla Espínola, Leticia Espinosa Del Barrio, Pedro Jesús Esteve Atiénzar, Carles García Cervera, David Francisco García Núñez, Francisco Garrido Navarro, Vicente Giner Galvañ, Angie Gómez Uranga, Javier Guzmán Martínez, Isidro Hernández Isasi, Lourdes Lajara Villar, Verónica Martínez Sempere, Juan Manuel Núñez Cruz, Sergio Palacios Fernández, Juan Jorge Peris García, Rafael Piñol Pleguezuelos, Andrea Riaño Pérez, José Miguel Seguí Ripoll, Azucena Sempere Mira, Philip Wikman-Jorgensen.

\section{H. Nuestra Señora del Prado. Talavera de la Reina (Toledo)}

Sonia Casallo Blanco, Jeffrey Oskar Magallanes Gamboa, Cristina Salazar Mosteiro, Andrea Silva Asiain.

\section{H. de Pozoblanco (Córdoba)}

José Nicolás Alcalá Pedrajas, Antonia Márquez García, Inés Vargas.

\section{H. G. U. de Elda (Alicante)}

Carmen Cortés Saavedra, Jennifer Fernández Gómez, Borja González López, María Soledad Hernández Garrido, Ana Isabel López Amorós, Santiago López Gil, Maria de los Reyes Pascual Pérez, Nuria Ramírez Perea, Andrea Torregrosa García.

\section{H. U. Infanta Cristina. Parla (Madrid)}

Juan Miguel Antón Santos, Ana Belén Barbero Barrera, Blanca Beamonte Vela, Coralia Bueno Muiño, Charo Burón Fernández, Ruth Calderón Hernáiz, Irene Casado López, José Manuel Casas Rojo, Andrés Cortés Troncoso, Pilar Cubo Romano, Francesco Deodati, Alejandro Estrada Santiago, Gonzalo García Casasola Sánchez, Elena García Guijarro, Francisco Javier García Sánchez, Pilar García de la Torre, Mayte de Guzmán García-Monge, Davide Luordo, María Mateos González, José A. Melero Bermejo, Cruz Pastor Valverde, José Luis Pérez Quero, Fernando Roque Rojas, Lorea Roteta García, Elena Sierra Gonzalo, Francisco Javier Teigell Muñoz, Juan Vicente de la Sota, Javier Villanueva Martínez.

\section{H. Santa Marina. Bilbao}

María Areses Manrique, Ainara Coduras Erdozain, Ane Labirua-Iturburu Ruiz.

\section{H. San Pedro. Logroño (La Rioja)}

Diana Alegre González, Irene Ariño Pérez de Zabalza, Sergio Arnedo Hernández, Jorge Collado Sáenz, Beatriz Dendariena, Marta Gómez del Mazo, Iratxe Martínez de Narvajas Urra, Sara Martínez Hernández, Estela Menendez Fernández, Jose Luís Peña Somovilla, Elisa Rabadán Pejenaute. 


\section{H. U. Son Llàtzer. Palma de Mallorca}

Andrés de la Peña Fernández, Almudena Hernández Milián.

\section{H. U. Ourense}

Raquel Fernández González, Amara Gonzalez Noya, Carlos Hernández Ceron, Isabel Izuzquiza Avanzini, Ana Latorre Diez, Pablo López Mato, Ana María Lorenzo Vizcaya, Daniel Peña Benítez, Milagros María Peña Zemsch, Lucía Pérez Expósito, Marta Pose Bar, Lara Rey González, Laura Rodrigo Lara.

\section{H. U. La Fe. Valencia}

Dafne Cabañero, María Calabuig Ballester, Pascual Císcar Fernández, Ricardo Gil Sánchez, Marta Jiménez Escrig, Cristina Marín Amela, Laura Parra Gómez, Carlos Puig Navarro, José Antonio Todolí Parra.

\section{H. de Mataró. Barcelona}

Raquel Aranega González, Ramon Boixeda, Javier Fernández Fernández, Carlos Lopera Mármol, Marta Parra Navarro, Ainhoa Rex Guzmán, Aleix Serrallonga Fustier.

\section{H. de Sagunto (Valencia)}

Enrique Rodilla Sala, Jose María Pascual Izuel, Zineb Karroud Zamrani.

\section{H. Alto Guadalquivir. Andújar (Jaén)}

Begoña Cortés Rodríguez.

\section{H. U. de Ferrol (A Coruña)}

Hortensia Alvarez Diaz, Tamara Dalama Lopez, Estefania Martul Pego, Carmen Mella Pérez, Ana Pazos Ferro, Sabela Sánchez Trigo, Dolores Suarez Sambade, Maria Trigas
Ferrin, Maria del Carmen Vázquez Friol, Laura Vilariño Maneiro.

\section{H. Infanta Margarita. Cabra (Córdoba)}

María Esther Guisado Espartero, Lorena Montero Rivas, Maria de la Sierra Navas Alcántara, Raimundo Tirado-Miranda.

\section{H. Público de Monforte de Lemos (Lugo)}

José López Castro, Manuel Lorenzo López Reboiro, Cristina Sardiña González.

\section{H. U. Virgen del Rocío. Sevilla}

Reyes Aparicio Santos, Máximo Bernabeu-Wittel, Santiago Rodríguez Suárez, María Nieto, Luis Giménez Miranda, Rosa María Gámez Mancera, Fátima Espinosa Torre, Carlos Hernandez Quiles, Concepción Conde Guzmán, Juan Delgado de la Cuesta, Jara Eloisa Ternero Vega, María del Carmen López Ríos, Pablo Díaz Jiménez, Bosco Baron Franco, Carlos Jiménez de Juan, Sonia Gutiérrez Rivero, Julia Lanseros Tenllado, Verónica Alfaro Lara, Aurora González Estrada.

\section{H. Marina Baixa. Villajoyosa (Alicante)}

Javier Ena, José Enrique Gómez Segado.

\section{A. U. de Salamanca}

Gloria María Alonso Claudio, Víctor Barreales Rodríguez, Cristina Carbonell Muñoz, Adela Carpio Pérez, María Victoria Coral Orbes, Daniel Encinas Sánchez, Sandra Inés Revuelta, Miguel Marcos Martín, José Ignacio Martín González, José Ángel Martín Oterino, Leticia Moralejo Alonso, Sonia Peña Balbuena, María Luisa Pérez García, Ana Ramon Prados, Beatriz Rodríguez-Alonso, Ángela Romero Alegría, Maria Sanchez Ledesma, Rosa Juana Tejera Pérez.

\section{H. General Defensa. Zaragoza}

Anyuli Gracia Gutiérrez, Leticia Esther Royo Trallero. 


\section{H. de Palamós (Girona)}

Ana Alberich Conesa, Mari Cruz Almendros Rivas, Miquel Hortos Alsina, José Marchena Romero, Anabel Martin-Urda Diez-Canseco.

\section{H. Comarcal de Blanes (Girona)}

Oriol Alonso Gisbert, Mercé Blázquez Llistosella, Pere Comas Casanova, Angels Garcia Flores, Anna Garcia Hinojo, Ana Inés Méndez Martínez, Maria del Carmen Nogales Nieves, Agnés Rivera Austrui, Alberto Zamora Cervantes.

\section{H. do Salnes. Vilagarcía de Arousa (Pontevedra)}

Vanesa Alende Castro, Ana María Baz Lomba, Ruth Brea Aparicio, Marta Fernández Morales, Jesús Manuel Fernández Villar, María Teresa López Monteagudo, Cristina Pérez García, Lorena Rodríguez Ferreira, Diana Sande Llovo, Maria Begoña Valle Feijoo.

\section{H. U. HM Montepríncipe}

José F. Varona Arche.

Acknowledgements We gratefully acknowledge all the investigators who participated in the SEMI-COVID-19 Registry. We also thank the SEMI-COVID-19 Registry Coordinating Centre, S and H Medical Science Service, for their quality control data, logistic and administrative support. We also thank CERCA Programme/Generalitat de Catalunya for institutional support.

\section{Declarations}

Conflict of interest The authors declare that they have no conflict of interest.

Ethical approval Our study was performed in accordance with the ethical standards of our institutional and with the 1964 Helsinki Declaration and its later amendments or comparable ethical standards.

Informed consent Written informed consent was waived by a central ethics committee, considering this an anonymized observational study in a pandemic situation.

\section{References}

1. C Bonanad S García-Blas FJ Tarazona-Santabalbina P Díez-Villanueva A Ayesta J Sanchis Forés MT Vidán-Austiz F Formiga A
Ariza-Solé M Martínez-Sellés 2020 (2020) Joint document of the section on geriatric cardiology of the Spanish Society of Cardiology and the Spanish Society of Geriatrics and Gerontology Rev Esp Cardiol 73569576

2. S Haque A Jawed N Akhter SA Dar F Khan RK Mandal MY Areeshi M Lohani M Wahid 2020 Acetylsalicylic acid (Aspirin): a potent medicine for preventing COVID-19 deaths caused by thrombosis and pulmonary embolism Eur Rev Med Pharmacol Sci 2492449245

3. AAR Mohamed-Hussein KME Aly MAA Ibrahim 2020 Should aspirin be used for prophylaxis of COVID-19-induced coagulopathy? Med Hypotheses 144109975

4. F Catella-Lawson MP Reilly SC Kapoor AJ Cucchiara S DeMarco B Tournier SN Vyas GA FitzGerald 2001 Cyclooxygenase inhibitors and the antiplatelet effects of aspirin N Engl J Med 3451809 1817

5. V Bianconi F Violi F Fallarino P Pignatelli A Sahebkar M Pirro 2020 Is Acetylsalicylic acid a safe and potentially useful choice for adult patients with COVID-19? Drugs 8013831396

6. HM Salah JL Mehta 2021 Meta-analysis of the effect of aspirin on mortality in COVID-19 Am J Cardiol 142158159

7. NM Alamdari S Afaghi FS Rahimi FE Tarki S Tavana A Zali M Fathi S Besharat L Bagheri F Pourmotahari SSN Irvani A Dabbagh SA Mousavi 2020 Mortality risk factors among hospitalized COVID-19 patients in a major referral center in Iran Tohoku J Exp Med 2527384

8. S Yuan P Chen H Li C Chen F Wang DW Wang 2020 Mortality and pre-hospitalization use of low-dose aspirin in COVID-19 patients with coronary artery disease J Cell Mol Med 251263 1273

9. JH Chow AK Khanna S Kethireddy D Yamane A Levine AM Jackson MT McCurdy A Tabatabai G Kumar P Park I Benjenk J Menaker N Ahmed E Glidewell E Presutto S Cain N Haridasa W Field JG Fowler D Trinh KN Johnson A Kaur A Lee K Sebastian A Ulrich S Pena R Carpenter S Sudhakar P Uppal BT Fedeles A Sachs L Dahbour W Teeter K Tanaka SM Galvagno DL Herr TM Scalea MA Mazzeffi 2020 Aspirin use is associated with decreased mechanical ventilation, ICU admission, and in-hospital mortality in hospitalized patients with COVID-19 Anesth Analg 132930941

10. M Rubio-Rivas X Corbella F Formiga E Menéndez Fernández MD Martín Escalante I Baños Fernández F Arnalich Fernández E Corral-Beamonte Del A Lalueza A Parra Virto E Roy Vallejo J Loureiro-Amigo AM Álvarez Suárez J Abadía-Otero M Navarro De La Chica R Estévez González A Hernández Milián M Areses Manrique JC Blázquez Encinar A González Noya R González Ferrer M Pérez Aguilera R Gil Sánchez J Millán Núñez-Cortés JM Casas-Rojo OBOT Semi-Covid-Network 2021 Risk categories in COVID-19 based on degrees of inflammation. Data on more than 17,000 patients from the Spanish SEMI-COVID-19 registry J Clin Med 102214

11. TF Osborne ZP Veigulis DM Arreola SM Mahajan E Röösli CM Curtin 2021 Association of mortality and aspirin prescription for COVID-19 patients at the Veterans Health Administration PLoS ONE 16 e 0246825

12. Sahai A, Bhandari R, Godwin M, McIntyre T, Chung MK, Iskandar JP, Kamran H, Hariri E, Aggarwal A, Burton R, Kalra A, Bartholomew JR, McCrae KR, Elbadawi A, Bena J, Svensson LG, Kapadia S, Cameron SJ (2021) Effect of aspirin on short-term outcomes in hospitalized patients with COVID-19. Vasc Med. 19: 1358863 X211012754.

13. M Haji Aghajani O Moradi H Amini H Azhdari Tehrani E Pourheidar MM Rabiei M Sistanizad 2021 Decreased in-hospital mortality associated with aspirin administration in hospitalized patients due to severe COVID-19 J Med Virol https://doi.org/10. 1002/jmv. 27053 
14. Q Liu N Huang A Li Y Zhou L Liang X Song Z Yang X Zhou 2021 Effect of low-dose aspirin on mortality and viral duration of the hospitalized adults with COVID-19 Medicine (Baltimore) 100 e 24544

15. ML Meizlish G Goshua Y Liu R Fine K Amin E Chang N DeFilippo C Keating Y Liu M Mankbadi D McManus SY Wang C Price RD Bona CI Ochoa Chaar HJ Chun AB Pine HM Rinder JM Siner DS Neuberg KA Owusu AI Lee 2021 Intermediate-dose anticoagulation, aspirin, and in-hospital mortality in COVID-19: a propensity score-matched analysis Am J Hematol 96471479

16. JW Martha R Pranata MA Lim A Wibowo MR Akbar 2021 Active prescription of low-dose aspirin during or prior to hospitalization and mortality in COVID-19-a systematic review and metaanalysis of adjusted effect estimates Int J Infect Dis 108612

17. R Sarzani F Giulietti C Pentima Di A Filipponi F Spannella 2020 Antagonizing the renin-angiotensin-aldosterone system in the era of COVID-19 Intern Emerg Med 15885887

18. D Onorato M Pucci G Carpene BM Henry F Sanchis-Gomar G Lippi 2021 Protective effects of statins Administration in
European and North American Patients Infected with COVID19: a meta-analysis Semin Thromb Hemost 47392399

19. AH Talasaz P Sadeghipour H Kakavand M Aghakouchakzadeh E Kordzadeh-Kermani BW Tassell Van A Gheymati H Ariannejad SH Hosseini S Jamalkhani M Sholzberg M Monreal D Jimenez G Piazza SA Parikh AJ Kirtane JW Eikelboom JM Connors BJ Hunt SV Konstantinides M Cushman JI Weitz GW Stone HM Krumholz GYH Lip SZ Goldhaber B Bikdeli 2021 Recent randomized trials of antithrombotic therapy for patients with COVID-19: JACC State-of-the-Art Review J Am Coll Cardiol 7719031921

20. MA Brookhart S Schneeweiss KJ Rothman RJ Glynn J Avorn T Stürmer 2006 Variable selection for propensity score models Am J Epidemiol 16311491156

Publisher's Note Springer Nature remains neutral with regard to jurisdictional claims in published maps and institutional affiliations.

\section{Authors and Affiliations}

Francesc Formiga ${ }^{1}$ (1) Manuel Rubio-Rivas ${ }^{1}$ - José María Mora-Luján ${ }^{1} \cdot$ Samara Campos Escudero $^{2}$.

Rosa Fernandez Madera Martinez ${ }^{3}$. Manuel Mendez-Bailón ${ }^{4}$. Pedro Durán-del Campo ${ }^{5}$. Andrea Riaño Pérez ${ }^{6}$. Francisco-Javier García-Sánchez ${ }^{7}$. José Nicolás Alcalá-Pedrajas ${ }^{8}$. Sergio Arnedo Hernández ${ }^{9}$. Almudena Hernández Milian ${ }^{10}$. Ana Latorre Díez ${ }^{11} \cdot$ Ricardo Gil Sánchez $^{12} \cdot$ Ramon Boixeda $^{13} \cdot$ Julio Vicente $^{14}$. Begoña Cortes ${ }^{15}$. Carmen Mella Pérez ${ }^{16}$. María Esther Guisado Espartero ${ }^{17}$. José López Castro ${ }^{18}$. Santiago Rodríguez Suárez ${ }^{19}$. Jose F. Varona ${ }^{20} \cdot$ Ricardo Gomez-Huelgas $^{21}$. Jose Manuel Ramos-Rincón ${ }^{22}$ • on behalf of the SEMI-COVID-19 SEMI-COVID-19 Network

Manuel Rubio-Rivas

mrubio@bellvitgehospital.cat

José María Mora-Luján

jmora@bellvitgehospital.cat

Samara Campos Escudero samara.campos1990@gmail.com

Rosa Fernandez Madera Martinez rosafmadera@hotmail.com

Manuel Mendez-Bailón manuel.mendez@salud.madrid.org

Pedro Durán-del Campo pedrodurandc@hotmail.com

Andrea Riaño Pérez andrea.riano.perez@gmail.com

Francisco-Javier García-Sánchez fcojgarciasanchez@gmail.com

José Nicolás Alcalá-Pedrajas jnalcala58@hotmail.com

Sergio Arnedo Hernández sarnedocascante@gmail.com

Almudena Hernández Milian nanamilian@hotmail.com

Ana Latorre Díez ana.latorre.diez@sergas.es

Ricardo Gil Sánchez gil_ricsan@gva.es
Ramon Boixeda

Rboixeda@csdm.cat

Julio Vicente

vicente.rodrigo.j@gmail.com

Begoña Cortes

begocortesrod@gmail.com

Carmen Mella Pérez

mellacarmen@gmail.com

María Esther Guisado Espartero guesm53@hotmail.com

José López Castro

jose.lopez.castro@sergas.es

Santiago Rodríguez Suárez santisanti84@hotmail.com

Jose F. Varona

jfvarona@hmhospitales.com

Ricardo Gomez-Huelgas ricardogomezhuelgas@hotmail.com

Jose Manuel Ramos-Rincón se.hmu@rsomar.esoj

1 Internal Medicine Department, Bellvitge University Hospital, Bellvitge Biomedical Research Institute-IDIBELL, University of Barcelona, L'Hospitalet de Llobregat, 08907 Barcelona, Spain

2 Internal Medicine Department, 12 de Octubre University Hospital, Madrid, Spain 
3 Internal Medicine Department, Cabueñes Hospital, Gijón, Asturias, Spain

4 Internal Medicine Department, Clinico San Carlos Hospital, Universidad Complutense, Madrid, Spain

5 Internal Medicine Department, Universitario Puerta de Hierro Hospital, Majadahonda, Madrid, Spain

6 Internal Medicine Department, H. U. S. Juan de Alicante, Alicante, Spain

7 Internal Medicine Department, Infanta Cristina University Hospital, Parla, Madrid, Spain

8 Internal Medicine Department, Pozoblanco Hospital, Córdoba, Spain

9 Internal Medicine Department, San Pedro de Logroño Hospital, Logroño, Spain

10 Internal Medicine Department, Son Llàtzer Universitario Hospital, Palma de Mallorca, Spain

11 Internal Medicine Department, Universitary Ourense Hospital, Ourense, Spain

12 Internal Medicine Department, Universitari I Politècnic La Fe Hospital, Valencia, Spain

13 Internal Medicine Department, Mataro Hospital, Barcelona, Mataro, Spain
14 Internal Medicine Department, Sagunto Hospital, Valencia, Spain

15 Internal Medicine Department,, Alto Guadalquivir Hospital, Jaén, Andújar, Spain

16 Internal Medicine Department, Clínico Universitario de Ferrol, A Coruña, Spain

17 Internal Medicine Department, Infanta Margarita de Cabra Hospital, Cordoba, Spain

18 Internal Medicine Department, Monforte de Lemos, Hospital, Lugo, Spain

19 Internal Medicine Department, Universitario Virgen del Rocío Hospital, Sevilla, Spain

20 Internal Medicine Department, Hospital Universitario HM Montepríncipe Hospital, Madrid, Spain

21 Internal Medicine Department, Regional University Hospital of Málaga, Biomedical Research Institute of Málaga (IBIMA), University of Málaga (UMA), Málaga, Spain

22 Internal Medicine Department, Miguel Hernandez University of Elche, Alicante, Spain 